Article

\title{
Long Noncoding RNA LncPGCR Mediated by TCF7L2 Regulates Primordial Germ Cell Formation in Chickens
}

\author{
Jingyi Jiang ${ }^{1}$, Chen Chen ${ }^{1}$, Shaoze Cheng ${ }^{1}$, Xia Yuan ${ }^{1}$, Jing Jin ${ }^{1}$, Chen Zhang ${ }^{1}$, Xiaolin Sun ${ }^{1}{ }^{\circledR}$, Jiuzhou Song ${ }^{2}{ }^{(0)}$, \\ Qisheng Zuo ${ }^{1}$, Yani Zhang ${ }^{1}$, Guohong Chen ${ }^{1}$ and Bichun $\mathrm{Li}^{1}{ }^{1} * \mathbb{1}$ \\ 1 Key Laboratory of Animal Breeding Reproduction and Molecular Design for Jiangsu Province, College of \\ Animal Science and Technology, Yangzhou University, Yangzhou 225009, Jiangsu, China; \\ jiangjy1127@163.com (J.J.); carolchen1996@163.com (C.C.); chengsz258@163.com (S.C.); \\ yuanxia0306@gmail.com (X.Y.); mx120170642@yzu.edu.cn (J.J.); m160647@yzu.edu.cn (C.Z.); \\ sunxiaolin19@sina.cn (X.S.); 006664@yzu.edu.cn (Q.Z.); ynzhang@yzu.edu.cn (Y.Z.); \\ ghchen@yzu.edu.cn (G.C.) \\ 2 Animal \& Avian Sciences, University of Maryland, College Park, MD 20741, USA; songj88@umd.edu \\ * Correspondence: yubcli@yzu.edu.cn
}

check for

updates

Citation: Jiang, J.; Chen, C.; Cheng, S.; Yuan, X.; Jin, J.; Zhang, C.; Sun, X.; Song, J.; Zuo, Q.; Zhang, Y.; et al. Long Noncoding RNA LncPGCR Mediated by TCF7L2 Regulates Primordial Germ Cell Formation in Chickens. Animals 2021, 11, 292. https://doi.org/10.3390/ani11020292

Academic Editors: Duy Ngoc Do and Prashanth N Suravajhala

Received: 28 December 2020

Accepted: 19 January 2021

Published: 24 January 2021

Publisher's Note: MDPI stays neutral with regard to jurisdictional claims in published maps and institutional affiliations.

Copyright: (c) 2021 by the authors. Licensee MDPI, Basel, Switzerland. This article is an open access article distributed under the terms and conditions of the Creative Commons Attribution (CC BY) license (https:// creativecommons.org/licenses/by/ $4.0 /)$.
Simple Summary: The potential of primordial germ cells (PGCs) for multidirectional differentiation, together with their unique regeneration ability, makes them one of the most promising seed cells in clinical medicine and tissue engineering research. However, not enough PGCs can be obtained to meet the demand, which limits their application. We defined a novel long noncoding RNA (lncRNA) mediated by epigenetics, which could activate the miR-6577-5p/Btrc pathway to promote the formation of PGCs. The technical system we have established is a useful tool to obtain sufficient PGCs for scientific research. Our study offers great theoretical and practical value in the production of transgenic animals or genomic imprinting in poultry. We believe that our study will help researchers in the fields of agricultural production, developmental biology, and cell biology.

Abstract: Although lncRNAs have been identified as playing critical roles in the development of germ cells, their potential involvement in the development of PGCs in chickens remains poorly understood. Differentially expressed lncRNAs (DELs) from previous RNA-seq of embryonic stem cells (ESCs), PGCs, and spermatogonial stem cells (SSCs) were analyzed by K-means clustering, from which a key candidate, IncRNA (lncRNA PGC regulator, LncPGCR) was obtained. We confirmed that LncPGCR plays a positive role in the development of PGCs by increasing the expression of the PGC marker gene (Cvh and C-kit), while downregulating the pluripotency-associated gene (Nanog) in vitro and in vivo. The activation and expression of $L n c P G C R$ are regulated by histone acetylation, and transcription factor TCF7L2. Mechanistically, a rescue assay was performed to further confirm that LncPGCR contributed to the development of PGCs by regulating the gga-miR-6577-5p/Btrc signaling pathway. Adsorption of gga-miR-6577-5p activated the WNT signaling cascade by relieving the gga-miR-6577-5p-dependent inhibition of Btrc expression. Taken together, our study discovered the growth-expedited role of $L n c P G C R$ in PGCs development, showing the potential $L n c P G C R / \mathrm{miR}$ $6577-5 \mathrm{p} /$ Btrc pathway. The results and findings provide a novel insight into the development of PGCs.

Keywords: chicken (Gallus gallus); LncRNA; primordial germ cell; TCF7L2; Btrc; ceRNA

\section{Introduction}

Chicken primordial germ cells (PGCs) would be invaluable for in vitro studies of aspects of chicken embryogenesis. The multidirectional differentiation potential and unique migration pathway are chicken PGCs' major biological properties, which can be applied to generate genetically modified poultry and conserve poultry's genetic resources [1]. The induction of paracrine signaling [2,3], inhibition of somatic fate [4], alteration of epigenetic 
marks [5], and maintenance of pluripotency [6] are important to the development of PGCs. Considerable effort has been devoted to maintaining and propagating chicken PGCs in vitro. However, the limited number of PGCs which can be obtained greatly limits their clinical application. Therefore, in-depth exploration is needed of the development mechanism of PGC, to overcome the limitations, and improve our technical ability to effectively cultivate or induce PGCs in vitro.

Since the discovery of PGCs, researchers have devoted extensive attention to the study of their developmental mechanism, which is a complex and sophisticated regulatory process, affected by many factors (Figure 1). Studies have shown that genes [4,7-9], signal pathways $[10,11]$, and growth factors $[12,13]$ all play important roles in the development of PGCs in mice, and research into their mechanism has gradually turned to the field of epigenetics [14]. The development of PGCs has been subjected to an epigenetic reprogramming process that is genome-wide $[15,16]$, including genome-wide DNA methylation erasure, chromosome inactivation, histone modification, and transposon silencing [17]. Current research on chickens shows only that genes such as Wnt5A, and FGF8 may regulate the formation of their primordial germ cells [18-20]. However, the efficiency of inducing the production of PGCs in vitro has not yet achieved the expected levels. Therefore, there is an urgent need for innovative exploration of the developmental mechanism of chicken PGCs from new directions.

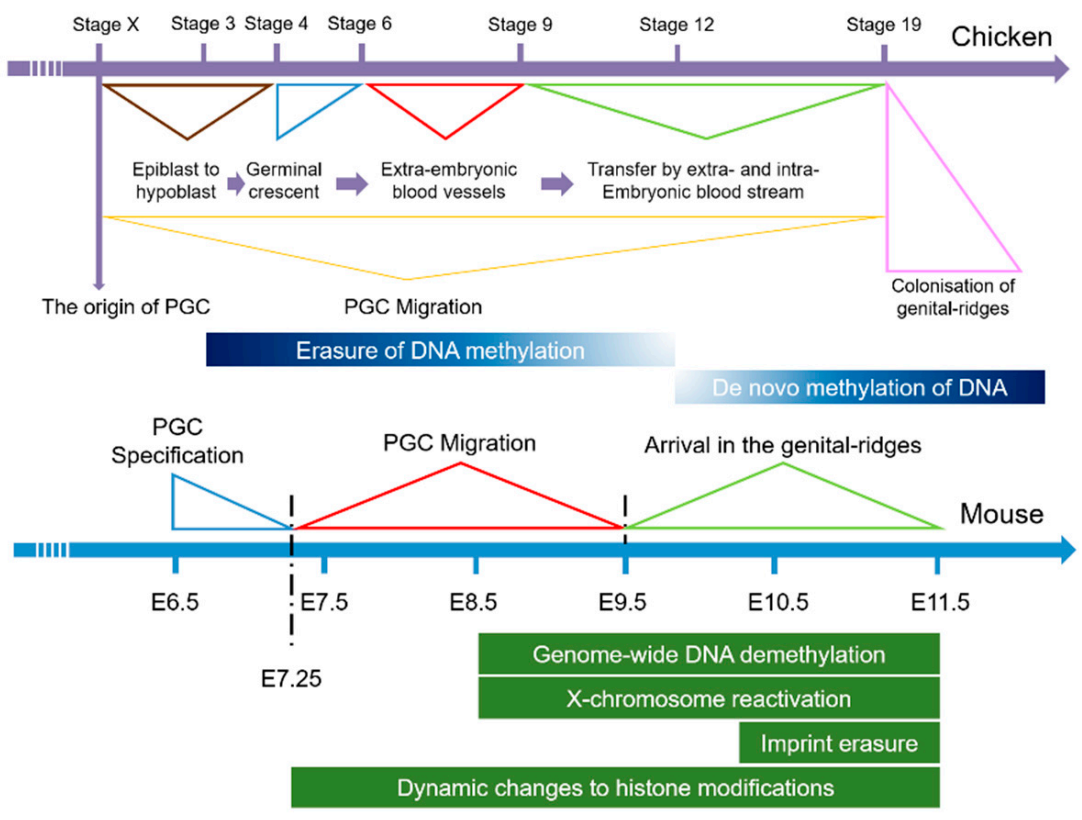

Figure 1. Development of mouse and chicken primordial germ cells.

The recent release of an ultra-high-throughput approach to the sequencing of RNA (RNA-seq) has paved the way for identification and annotation of numerous long noncoding RNAs in the chicken genome [21]. Also, a quantity of binding sites for PGC marker genes (Blimp1/Prdm1) were found to be related to lncRNA in mouse PGCs [7]. Therefore, lncRNAs have recently come to the forefront as functional molecules involved in cell differentiation by interacting with microRNA [22], recruiting proteins as scaffolds to form complexes [23], and trapping transcription factors [24,25]. While different expression positions of LncRNA exhibit distinct molecular mechanisms [26,27], the specific regulatory mechanism of LncRNA during the differentiation of ESCs into PGCs remains unclear.

Here, we provide evidence that LncRNAs intimately regulate PGC development through a novel network of functional interaction between lncRNAs and miRNAs. In this study, we have identified a novel PGC-specific lncRNA (LncPGCR, TCONS_00948124) in RNA-seq analysis results of genes up-regulated in PGCs. We found that increased LncPGCR levels were activated by the transcription factor TCF7L2, and, histone acetylation. 
Moreover, $L n c P G C R$ was also shown to regulate the gga-miR-6577-5p targeted gene Btrc by functioning as a competitive endogenous RNA (ceRNA) for gga-miR-6577-5p, thereby promoting the expression of Btrc and facilitating lncRNA-directed PGC development.

\section{Materials and Methods}

\subsection{Ethics Approval}

Animal experiments were approved by the Institutional Animal Care and Use Committee of the Yangzhou University Animal Experiments Ethics Committee (permit number: SYXK [Su] IACUC 2012-0029). All experimental procedures were performed in accordance with the Regulations for the Administration of Affairs Concerning Experimental Animals approved by the State Council of the People's Republic of China.

\subsection{Antibody and Reagent}

Commercially available antibodies were used: anti-DDX4/Cvh (ab13840, Abcam, Cambridge, UK); Goat Anti-Mouse IgG (Cy3 labeled, Bio-Synthesis, Inc., Louisville, KY, USA; dilution ratio:1:100); anti-NANOG and anti-OCT4 (Abcam, dilution ratio 1:100); anti-SSEA (BioLegend, San Diego, CA, USA, dilution ratio 1:100); anti-integrin $\alpha 6$ and anti-integrin $\beta 1$ (BioLegend, dilution ratio 1:100).

Dulbecco's modified Eagle medium (DMEM, 41965062) and fetal bovine serum (FBS, 10100-147) from Gibco (Grand Island, NY, USA); FuGENE ${ }^{\circledR} \mathrm{HD}$ (E2311) and DualLuciferase ${ }^{\circledR}$ Reporter Assay System from Promega (Madison, WI, USA); Leukemia Inhibit Factor from mouse (mLIF, L5158), Fibroblast Growth Factor-Basic human (bFGF, F0291), Stem Cell Human (SCF, S7901); TSA from Sigma (St. Louis, MO, USA); PARISTM from Ambion (Austin, TX, USA); retinoic acid (R2625) from Sigma.

\subsection{Experimental Animals}

A total of 180 fertilized Rugao yellow eggs were purchased from the Poultry Research Institute of the Chinese Academy of Agricultural Sciences (Yangzhou, China). All eggs were incubated at $37^{\circ} \mathrm{C}$ and $75 \%$ relative humidity. Tissues of heart, liver, lung, glandular stomach, gizzard, testis, and ovary obtained from 3 adult chickens were harvested for the organ-specific expression analysis.

\subsection{Cell Culture and Plasmids}

In vitro isolation of ESCs required 60 freshly fertilized eggs. ESCs were isolated using blastoderm cells in the embryonic region of stage $X$ fertilized eggs. The isolated blastoderm cells were cultured in DMEM containing $10 \%$ FBS, $2 \%$ Chicken serum, $10 \mathrm{ng} / \mathrm{mL}$ bFGF, $1000 \mathrm{IU} / \mathrm{mL} \mathrm{LIF}$, and $5 \mathrm{ng} / \mathrm{mL} \mathrm{SCF}$ at $37^{\circ} \mathrm{C}$, and $5 \% \mathrm{CO}_{2}$ saturated humidity for $24 \mathrm{~h}$ and then subjected to differential purification of ESC.

Sixty freshly fertilized eggs incubated for 4.5 days were harvested to obtain genital ridges for isolation of PGC. Chicken embryo genital ridges were digested with $0.25 \%$ trypsin and $0.05 \%$ EDTA for $8 \mathrm{~min}$. The PGCs were purified by repeated differential adherence techniques. The first purification selection was performed after cell culturing for $40 \mathrm{~min}$, and non-adherent cells were transferred to a new culture dish. The supernatant was further cultured at $37^{\circ} \mathrm{C}$ and $5 \% \mathrm{CO}_{2}$ saturated humidity.

Sixty freshly fertilized eggs incubated to 18.5 days were harvested to obtain testes for the isolation of SSC. The testis tissue was digested with collagenase for $30 \mathrm{~min}$, and then with $0.25 \%$ trypsin and $0.05 \%$ EDTA for $8 \mathrm{~min}$. The SSCs were purified by the repeated differential adherence technique. The first purification selection was performed after cell culturing for $40 \mathrm{~min}$, and non-adherent cells were transferred to a new culture dish. The supernatant was further cultured at $37^{\circ} \mathrm{C}$ and $5 \% \mathrm{CO}_{2}$ saturated humidity.

We prepared plasmid pcDNA3.0-LncPGCR. RNAi sequence: LncPGCR: sh-1 targets GGATCTGGTTTAATCCCATCG; sh-2 targets GCCATTTCTCATCAGAGTAAG; sh-3 targets GGATCTGGTTTAATCCCATCG. The sequence of miRNA-mimic amplification primers is shown in Table 1. 
Table 1. pri-miR primer sequences.

\begin{tabular}{cc}
\hline Name & Sequence $\left(5^{\prime}\right.$ to $\left.\mathbf{3}^{\prime}\right)$ \\
\hline mir-6577-5p-F & CCGGAATTCGGCTGCTAAGGTATAGCACAGCTGTG \\
mir-6577-5p-R & CCGGGATCCCCTTACCCAGCAGAGCAACTCAG \\
\hline
\end{tabular}

\subsection{FACS and RNA-seq}

ESCs that had been induced for 4 days were labeled by CVH. The Fuzzy C-Means algorithm (FCM) was used to detect the number of PGCs. Finally, data were analyzed on a Flow cytometer (Becton Dickinson, San Jose, CA, USA) with the FlowJo program.

Using FACSAria SORP to sort ESCs, PGCs and SSCs were labeled with antibodies to ensure that high-purity cells were obtained. Among them, Nanog and Oct4 stem cell surface-specific antigens and molecular markers were used to label ESC, tyrosine kinase receptor C-kit and SSEA-1 to label PGC, and spermatogonial stem cell surface markers integrin $\alpha 6$ and integrin $\beta 1$ to label SSC. Total RNA was extracted from ESCs, PGCs, and SSCs, using the TRNzol reagent (Invitrogen, Carlsbad, CA, USA) according to the manufacturer's instructions. RNA degradation and contamination were monitored on $1 \%$ agarose gels. RNA purity was checked using the NanoPhotometer ${ }^{\circledR}$ spectrophotometer (IMPLEN, CA, USA). RNA concentration was measured using Qubit ${ }^{\circledR}$ RNA Assay Kit in Qubit ${ }^{\circledR}$ 2.0 Fluorometer (Life Technologies, Carlsbad, CA, USA). RNA integrity was assessed using the RNA Nano 6000 Assay Kit of the Bioanalyzer 2100 system (Agilent Technologies, Santa Clara, CA, USA). Sequencing library construction was conducted by using the TruSeq PE Cluster Kit v3-cBot-HS (Illumina, San Diego, CA, USA) according to the manufacturer's instructions. The libraries were sequenced on an Illumina Hiseq 2000 platform. All sequence data are available in SRA with the Accession No. SRR12145635, SRR12145634, and SRR12145633.

\subsection{Isolation of Nuclear and Cytoplasmic RNA}

Operations were performed according to the manufacturer's protocols of PARISTM system Protein and RNA Isolation System: “add $500 \mu \mathrm{L}$ Cell Disruption Buffer to every $10^{7}$ PGCs cells, and incubate on ice for $10 \mathrm{~min}$; After centrifugation at $500 \times \mathrm{g}$ for $5 \mathrm{~min}$ at $4{ }^{\circ} \mathrm{C}$, the supernatant is cytoplasm and the precipitate is the nucleus." To assess any cross-contamination between cytoplasmic and nuclear fractions, the levels of GAPDH (a specific cytoplasmic protein marker) and U1 (a nuclear protein marker) were examined in the two fractions.

\subsection{Chicken Embryo Vascular Injection}

Eggs were swabbed with alcohol swabs before injection using tweezers to open a round hole with a diameter of 1-1.5 cm. After finding the position of the embryo under the stereomicroscope, ophthalmic tweezers were used to gently lift the outer membrane to expose the embryonic blood vessel. Using a micropipette, the lentiviral vector was filled with a final polybrene concentration of $8 \mathrm{ng} / \mu \mathrm{L}$ or the pcDNA 3.0-PGCR vector wrapped with PEI into glass injection needles and injected into the blood vessels of the chicken embryos. After the injection, $20 \mu \mathrm{L}$ of penicillin was added to the injection site and then cross-sealed with medical tape to continue incubation.

\section{8. $q R T-P C R$}

Total RNA was extracted using Trizol (TIANGEN, Beijing, China) and reversetranscribed into cDNA with the Quantscript RT Kit (TIANGEN). Gene expression was determined using an ABI PRISM 7500 fluorescent quantitative PCR instrument (Applied Biosystems, Carlsbad, CA, USA). qRT-PCR primers are shown in Table 2, with the internal reference gene: $\beta$-actin. 
Table 2. Primer information for qRT-PCR.

\begin{tabular}{cc}
\hline Gene & Primer Sequence \\
\hline \multirow{2}{*}{-actin } & F: CAGCCATCTTTCTTGGGTAT \\
& R: CTGTGATCTCCTTCTGCATCC \\
Gapdh & F: TCAAATGGGCAGATGCAGGT \\
& R: TCAGCAGCAGCCTTCACTAC \\
U1 & F: ACATGGTGTACAACAAGCGC \\
& R: CTCACCGCTCATCGTATCGG \\
LncPGCR & F: TGGATGGTCAAAGGGATGCC \\
& R: ATGAGAAATGGCTGTGGGGG \\
Cvh & F: CCACGGCTATTTCACACCTCTG \\
& R: GCTCTTGGCAAGCATCCGTA \\
C-kit & F: GCGAACTTCACCTTACCCGATTA \\
& R: TGTCATTGCCGAGCATATCCA \\
Nanog & F: TGGTTTCAGAACCAACGAATGAAG \\
& R: TGCACTGGTCACAGCCTGAAG \\
Btrc & F: TCAAATGGGCAGATGCAGGT \\
& R: TCAGCAGCAGCCTTCACTAC \\
gga-mir-6577-5p & F: acggcgcgCATCTTCTACTCTGTTTCTTCT \\
& R: CAGTGCGTGTCGTGGAGT \\
\hline
\end{tabular}

\subsection{Indirect Immunoinfluscent Assay (IFA)}

ESCs that had been induced for 4 days were fixed with $4 \%$ paraformaldehyde for $30 \mathrm{~min}$ and then treated with $0.5 \%$ TritonX-100 for $15 \mathrm{~min}$. After blocking with $10 \%$ fetal bovine serum at $37^{\circ} \mathrm{C}$ for $2 \mathrm{~h}$, the cells were incubated in anti-CVH (1:500, Abcam) for $12 \mathrm{~h}$ at $4{ }^{\circ} \mathrm{C}$; After multiple washes by PBST, cells were incubated with corresponding secondary antibodies (1:400, Abcam) for $2 \mathrm{~h}$ at room temperature. After DAPI (5 ng/uL) staining, slides were plated with glycerin, and the image was sealed. The FV1000 laser scanning confocal microscope (Olympus, Tokyo, Japan) was used to observe the samples.

\subsection{Dual-Luciferase Assays}

The amplified promoter of $L n c P G C R$ and different deletion fragments of the $L n c P G C R$ promoter were subcloned into the firefly plasmids in the pGL3Basic luciferase vector (Promega). Different length PCR amplification primers of the LncPGCR promoter region are shown in Supplementary Table S1. TCF7L2 binding site mutation primers are shown in Supplementary Table S2. Finally, a Dual-Luciferase Reporter Assay System (Promega) was used to evaluate luciferase activity. Each procedure of these experiments was repeated independently three times.

\subsection{Data Analysis}

Relative gene expression was calculated using the $2^{-\Delta \Delta \mathrm{Ct}}$ method after PCR [28]. All experiments were performed in triplicate, and the data are expressed as mean \pm standard error. Significant differences between the groups were determined with two-sample $t$ tests in SPSS 18.0. $\left(^{*}, p<0.05\right.$, significant difference. ${ }^{* *}, p<0.01$, extremely significant difference). GraphPad Prism7 software (GraphPad Software, San Diego, CA, USA) was used for mapping.

\section{Results}

\subsection{Searching for LncRNAs Involved in the Development of PGCs}

To identify lncRNAs involved in the regulation of PGCs development, we performed an in-depth analysis of differentially expressed lncRNAs by RNA-seq (Figure 2A,B). A comparison of the lncRNA expression profiles in chicken ESCs, PGCs, and SSCs showed that 15 lncRNAs were differentially expressed in PGCs (Table 3, Table S3). We focused on LncRNA (LncPGCR, TCONS_00948124), since it was strongly related to germ cell differentiation and because, based on computational prediction algorithms, there were potential interactions of 
LncPGCR with several miRNAs including gga-miR-6577-5p. Then, we evaluated the level of LncPGCR expression in ESCs, PGCs, and SSCs by qRT-PCR, and results indicated that LncPGCR was also up-regulated in PGCs $(p<0.01)$ (Figure 2C). Chicken tissue expression profiling revealed that $L n c P G C R$ was highly expressed in gonads $(p<0.01)$ (Figure 2D), suggesting that $L n c P G C R$ was likely to be involved in PGC development.

A

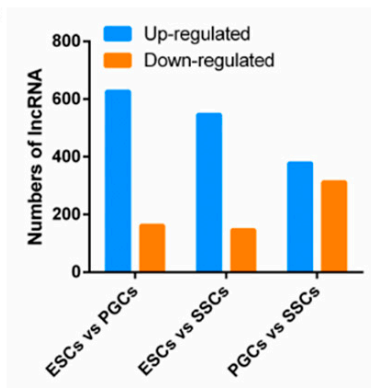

D



B

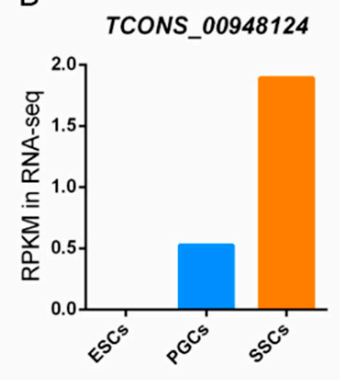

C

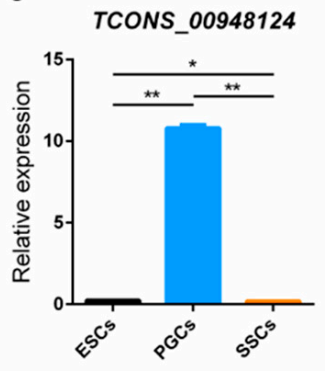

$E$

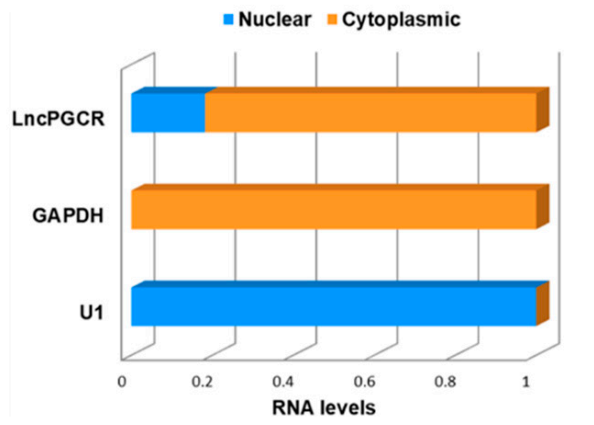

Figure 2. $L n c P G C R$, a PGC specific LncRNA in Chicken, is mainly expressed in the cytoplasm. (A) LncRNAs differentially expressed in ESCs vs PGCs, ESCs vs SSCs, and PGCs vs SSCs as examined by RNA-seq. (B) RNA-seq measurement showing the transcript expression levels of LncPGCR in ESCs, PGCs, and SSCs. (C) Validation of mRNA expression levels of LncPGCR by qRT-PCR. Values are means \pm SEM. $n=3$. (D) Relative expression levels of $L n c P G C R$ in adult chicken tissues as measured by qRT-PCR after normalization to $\beta$-actin. ${ }^{*} p<0.05$, and ${ }^{* *} p<0.01$. Values are means \pm SEM. $n=3$. (E) Relative expression levels of LncPGCR in the nucleus and cytoplasm of PGCs as measured by qRT-PCR after normalization to $\beta$-actin. ${ }^{*} p<0.05$, and ${ }^{* *} p<0.01$. Values are means \pm SEM. $n=3$.

We found that $L n c P G C R$ was expressed in both the nucleus and the cytoplasm of the PGCs, but the expression level in the cytoplasm was much higher than that in the nucleus, which indicated that the mechanism of LncPGCR action mainly existed in the post-transcriptional level (Figure 2E). These data demonstrated that LncPGCR is highly expressed in the cytoplasm of PGCs and might be critical to the development of PGCs in chickens.

\subsection{LncPGCR Promotes Development of PGCs In Vitro and In Vivo}

To define the function of $\operatorname{LncPGCR}$ on PGC development, we transfected the $\operatorname{LncPGCR}$ overexpression/knockdown vectors based on the retinoic acid (RA) induction model and observed the morphology of ESC cells (Figure 3A,B). After the over-expression of LncPGCR, we observed a large number of embryonic bodies and PGC-like structures in ESCs after 2-4 days of RA induction. However, knockdown $L n c P G C R$ triggers a significant decline in PGC-like development (Figure 3C).

Indirect immunoinfluscent assay and flow cytometry analysis confirmed that $L n c P G C R$ promotes the development of PGCs. Specifically, the percentage of Cvh $h^{+}$PGCs at $4 \mathrm{~d}$ induction in the RA model was significantly higher in the LncPGCR overexpression group than in the control group or the $L n c P G C R$ interference group (Figure 3D,E). qRT-PCR results 
indicated that overexpression of $\operatorname{LncPGCR}$ accelerates the down-regulation of the totipotent gene Nanog to promote cell differentiation $(p<0.05)$ (Supplementary Figure S1A). At the same time, overexpression of $L n c P G C R$ promotes the expression of the PGC marker genes $C v h$ and $C$-kit $(p<0.01)$. In contrast, LncPGCR knockdown led to significant increases in Nanog expression, and the expression of Cvh and C-kit showed significant downregulation.

Table 3. Differences in Specific Expression Related to Germ Cell Differentiation in PGCs lncRNA.

\begin{tabular}{ccc}
\hline LncRNA & Target Gene & KEGG \\
\hline TCONS_00612668 & BCL9L & - \\
TCONS_00765266 & Lef-1 & Wnt signaling pathway, Adherens junction, \\
Melanogenesis
\end{tabular}

To further verify the function of $L n c P G C R$ in the development of PGC, we injected the PGCR-sh2 lentiviral vector and pcDNA3.0-PGCR vector into the E2.5d chicken embryonic blood vessels. qRT-PCR results showed that after knocking down $L n c P G C R$, the expression of Nanog was increased $(p<0.01)$, while the expression of Cvh and C-kit were decreased $(p<0.01)$ (Supplementary Figure S1B). Flow cytometry analysis confirmed that the development of PGCs was suppressed after the knockdown of $\operatorname{LncPGCR}(1 \% \pm 0.19)$ (Supplementary Figure S1C), which was consistent with the results of induction in vitro. Hence, we confirmed that $L n c P G C R$ promotes the development of PGCs.

\subsection{Deacetylation Downregulate LncPGCR Expression}

To elucidate the mechanisms regulating LncPGCR expression in the PGC development, we cloned the promoter of $L n c P G C R$ and replaced the CMV promoter of pEGFP-N1 (Figure 4A). We ligated a series of promoter fragments into the pGL3-basic vector and transfected into DF1 cells to identify the core region of the LncPGCR promoter with a dual luciferase reporter system (Figure 4B). The dual-luciferase assay revealed that the $-1033 \sim-661$ bp region was the core active region of the LncPGCR promoter (Figure 4C).

Epigenetic determinants are known to establish and maintain specific patterns of transcription through modulation of DNA methylation and posttranslational modifications of core histones during development [12,29-31]. Histone deacetylase (HDAC) is a class of enzymes that removes acetyl groups from a histone, allowing the histones to wrap the DNA more tightly [29]. TSA can bind to HDAC, inhibit histone deacetylation, and promote gene expression. To assess whether epigenetic factors affected LncPGCR expression, we add TSA $(1 \mu \mathrm{mol} / \mathrm{L})$ to DF-1 cells transfected with $\mathrm{pGL} / 1614$. The dual-luciferase assay verified that TSA significantly increase the LncPGCR promoter activity $(p<0.01)$ (Figure $4 \mathrm{D})$. Together, our results indicated that deacetylation are involved in the downregulation of LncPGCR. 
A

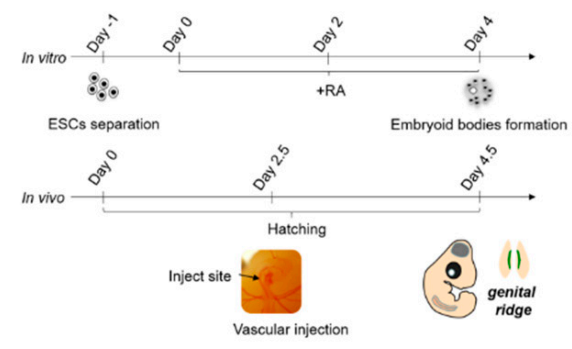

B

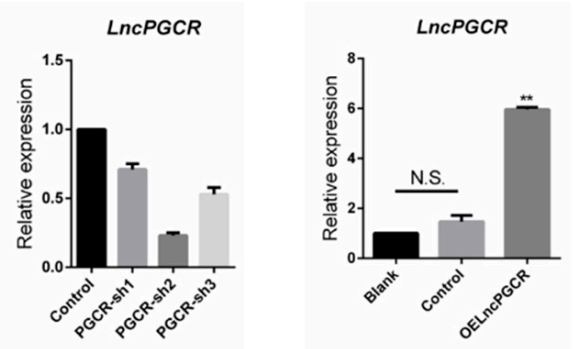

C

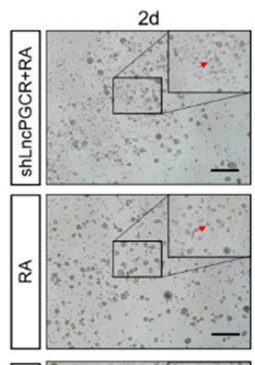

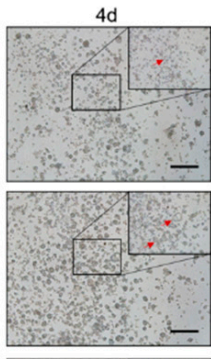

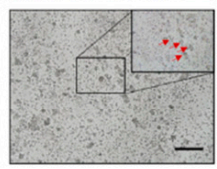

D

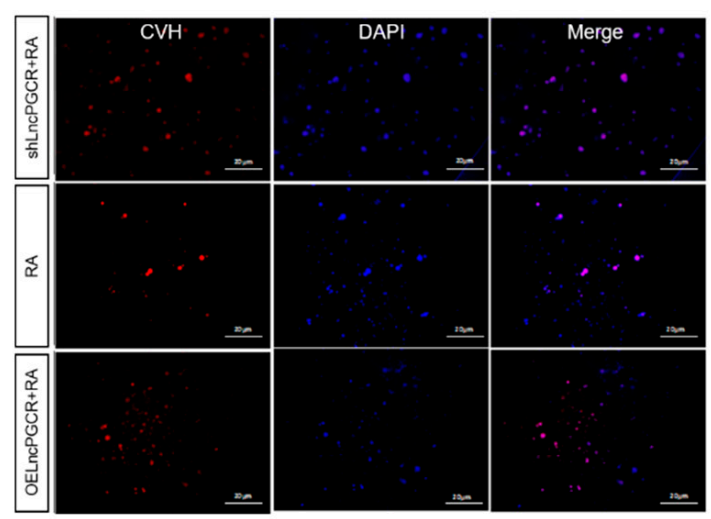

$\mathrm{E}$

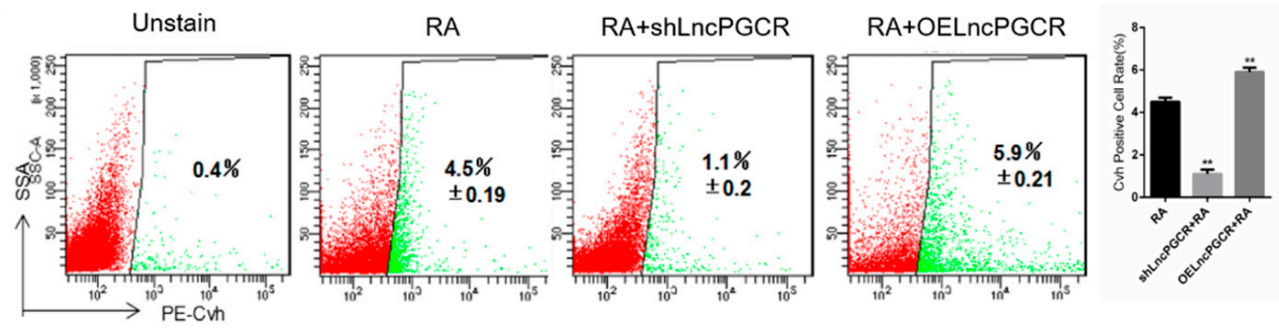

Figure 3. Ectopically expressed LncPGCR promotes PGCs formation in vitro and in vivo. (A) Schematic diagram of $L n c P G C R$ function verification in vivo and in vitro. (B) Activity verification of $L n c P G C R$ overexpression and knockdown expression vectors. (C) Morphological changes of cells after overexpression or knockdown of $L n c P G C R$ in the RA induction model. (D) Indirect immunoinfluscent analysis of Cvh (red) on day 4 after overexpression or knockdown with LncPGCR in the RA induction model in vitro. Scale bar: $20 \mu \mathrm{m}$. (E) The proportion of d4 PGC-like cells (Cvh+) was detected by flow cytometry analysis.

\subsection{Transcription Factor TCF7L2 Regulates the Expression of LncPGCR}

Transcription factors (TFs) play a pivotal role in the regulation of gene expression [30,31]. To further explore the regulators of the LncPGCR promoter, we predicted the transcription factors which might bind with the promoters of LncPGCR by the JASPAR databases. There are binding sites for transcription factors STAT1, TCF7L2, and Sox3 in the core region of the LncPGCR promoter, and TCF7L2 had the highest score (Figure 4E). Moreover, the dualluciferase assay revealed that mutation of the TCF7L2 binding site significantly reduced the LncPGCR promoter activity $(p<0.01)$, indicating that TCF7L2 is a positive regulator of the LncPGCR promoter (Figure 4F). Interestingly, the transcription factor TCF7L2 was upregulated during the induction of ESCs into PGCs in vitro, consistent with the expression trend of LncPGCR (Figure 4G). All these results indicated that TCF7L2 served as a positive regulator of $L n c P G C R$. 

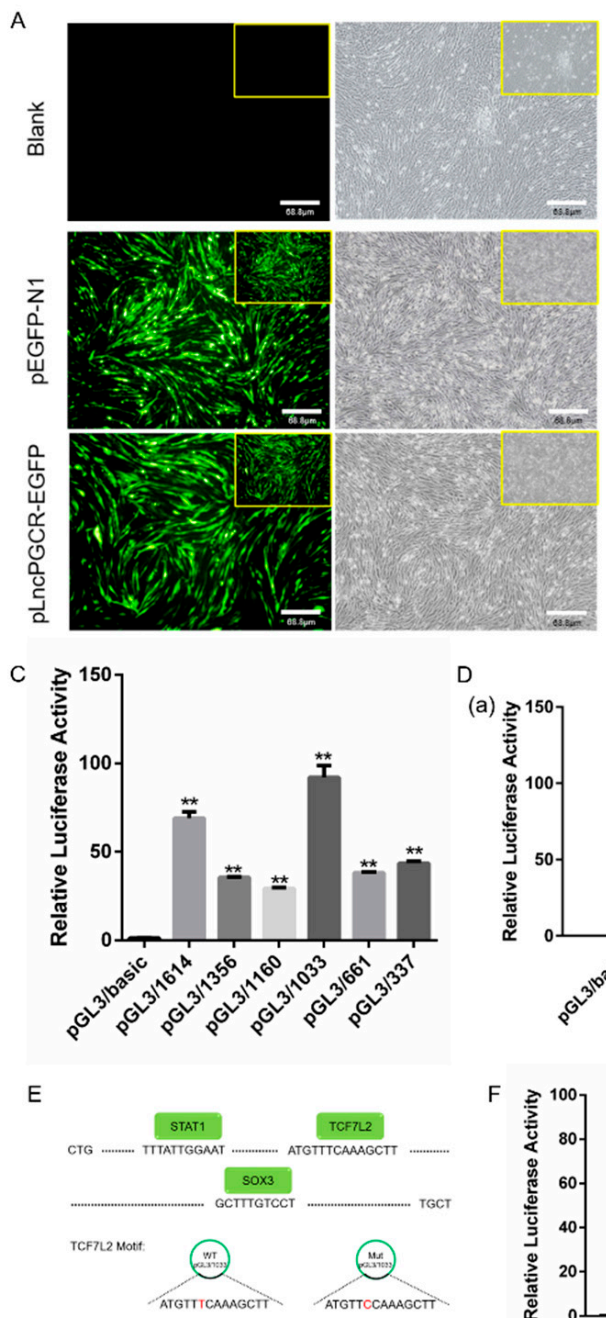
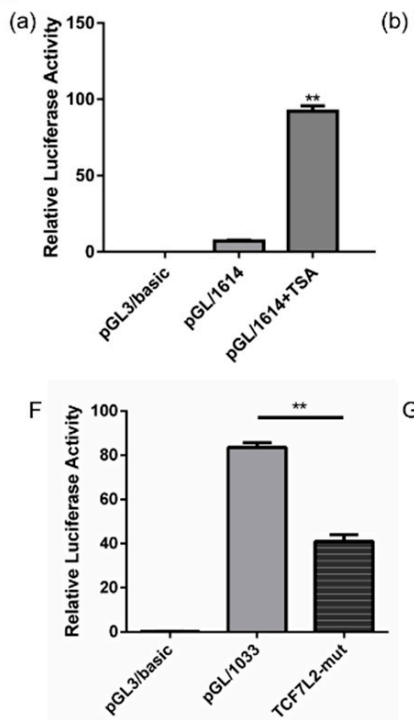

(b)
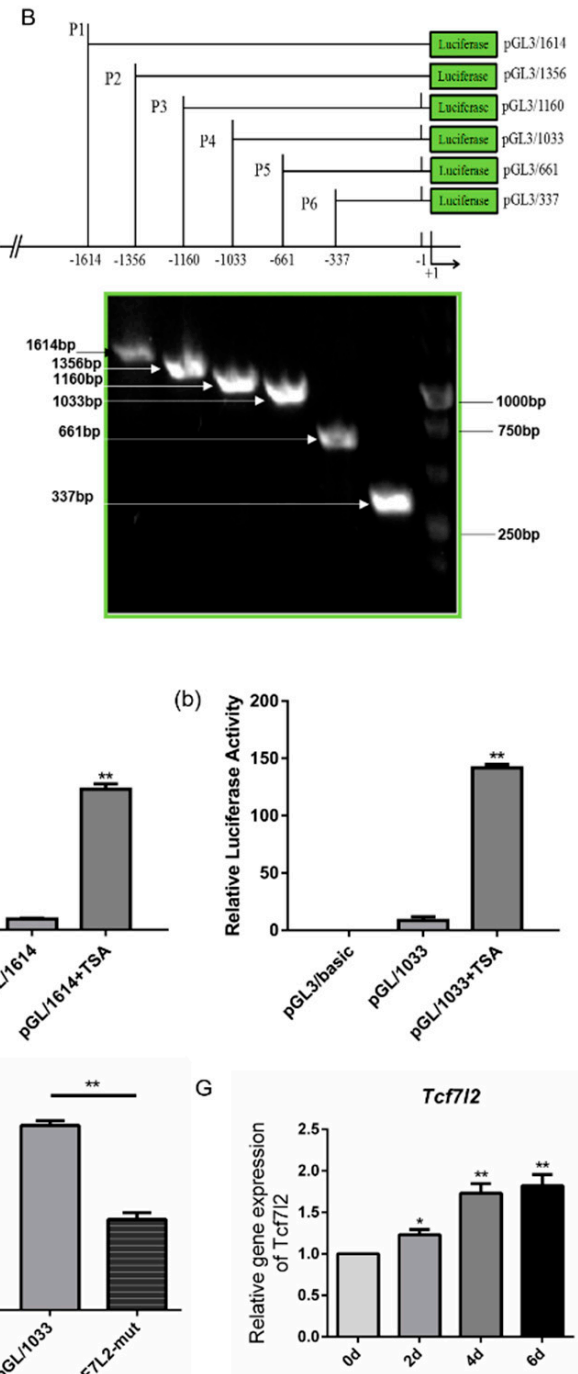

Figure 4. Histone acetylation, and TCF7L2 regulate the expression of LncPGCR. (A) Qualitative activity detection of $L n c P G C R$ promoter long fragment $(100 \times)$. Scale bar: $68.8 \mu \mathrm{m}$. (B) Schematic diagram of vector construction for $L n c P G C R$ promoter. (C) Dual luciferase analysis of the core region of LncPGCR' promoter. * $p<0.05$, and ${ }^{* *} p<0.01$ compared with the control pGL3/BASIC vector. Values are means \pm SEM. $n=3$. (D) Levels of LncPGCR promoter activity in DF1 cells after adding TSA co-transfected with pGL3/1614(a) or pGL3/1033(b). (E) Top: the location of STAT1, TCF7L2, and SOX3 in the core region of the LncPGCR promoter; Bottom:diagram of TCF7L2 transcription factor binding site mutation. (F) Levels of LncPGCR promoter activity in DF1 cells after mutation of the TCF7L2 binding site. ${ }^{*} p<0.05$, and ${ }^{* *} p<0.01$ compared with the control pGL3/BASIC vector. Values are means \pm SEM. $n=3$. (G) qRT-PCR of Tcf712 expression during the induction of ESCs into PGCs in vitro. Values are means \pm SEM. $n=3$.

\subsection{LncPGCR Functions as a ceRNA for gga-miR-6577-5p}

Salmena [32] proposed a competitive endogenous RNA (ceRNA) hypothesis, that mRNA, transcribed pseudogenes, and lncRNA act as natural miRNA sponges by sharing one or more miRNA response elements in a large-scale regulatory network to inhibit miRNA function, thereby affecting the occurrence and development of diseases. To test if $L n c P G C R$ stimulates development of PGCs by modulating the miRNA function, we evaluated the miRNA binding sites on LncPGCR using DIANA TOOLS and found 91 miRNA binding sites. Cis-tether prediction found that the target gene of $L n c P G C R$ is Btrc (Table 3). Therefore, we used miRDB to predict the miRNAs that have binding sites for 
Btrc in chicken and found 71 miRNAs. Three miRNAs (gga-mir-6577-5p, gga-mir-6625-5p, and gga-mir-3539) with binding sites to Btrc and LncPGCR were screened by Venn analysis (Figure 5A). Based on their bioinformatics scores, we chose gga-miR-6577-5p to verify the assumption (Figure 5B). qRT-PCR results showed that $L n c P G C R$ promoted the expression of Btrc, while gga-miR-6577-5p inhibited its expression (Figure 5C,D), confirming that Btrc is a common target gene of $L n c P G C R$ and gga-miR-6577-5p. Strikingly, the co-transfection of Mimic-6577-5p and pcDNA3.0-LncPGCR reversed the transcription-inhibitory role of gga-miR-6577-5p (Figure 5E).

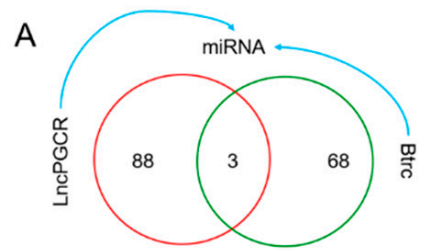

C

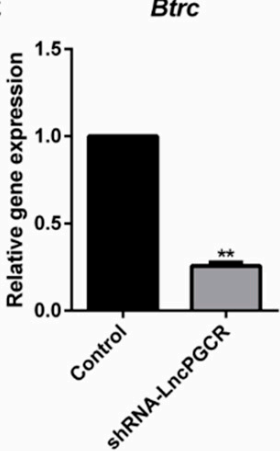

B

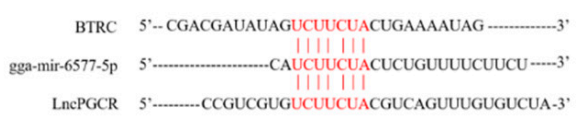

Btrc

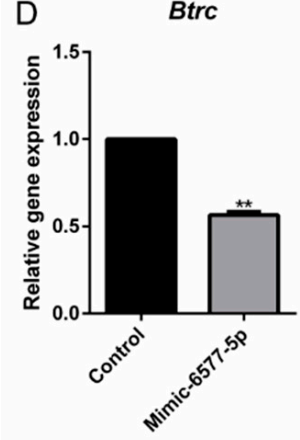

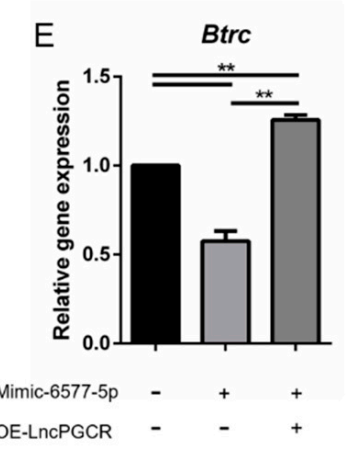

$\mathrm{F}$
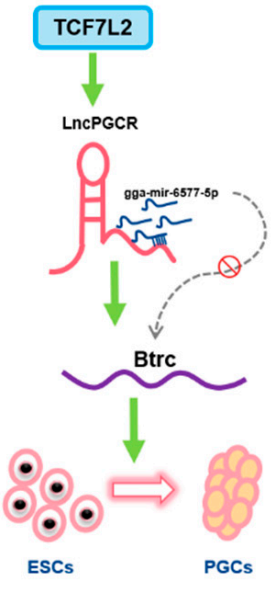

Figure 5. LncPGCR and gga-miR-6577-5p regulate PGC development through their target gene Btrc. (A) Screen potential miRNA targets of $L n c P G C R$ and Btrc by analyzing crosstalk results of RNA-seq and miRDB. (B) Sequence alignment of gga-miR-6577-5p with the putative binding sites of LncPGCR and Btrc. (C) LncPGCR can up-regulate Btrc expression as measured by qRT-PCR. ${ }^{*} p<0.05$, and ${ }^{* *} p<0.01$ compared with control. Values are means \pm SEM. $n=3$. (D) gga-miR6577-5p can inhibit Btrc expression as measured by qRT-PCR. ${ }^{*} p<0.05$, and ${ }^{* *} p<0.01$ compared with control. Values are means \pm SEM. $n=3$. (E) Up-regulated gga-miR-6577-5p in DF1 cells, which stably over-expressed LncPGCR, largely reversed the favorable effects of $\operatorname{LncPGCR}$ on Btrc expression. ${ }^{*} p<0.05$, and ${ }^{* *} p<0.01$ compared with control. Values are means \pm SEM. $n=3$. (F) Model proposed to explain the influence of LncPGCR upon the PGCs development.

\section{Discussion}

In this present study, we identified several LncRNAs that are aberrantly expressed in PGCs. Among them, LncPGCR was the most upregulated in PGCs. Here, we showed that $L n c P G C R$ was highly expressed in PGCs and gonad tissues, and high LncPGCR expression was closely associated with the development of PGCs. We also confirmed that the overexpression of $L n c P G C R$ can promote PGC development, both in vivo and in vitro, and that TCF7L2 mediated upregulation of LncPGCR. Mechanistically, we showed that $L n c P G C R$ directly interacted with gga-miR-6577-5p at the recognized sites. LncPGCR exerted its function on PGCs in large part due to its role as a ceRNA for gga-miR-6577-5p, and subsequently to initiate the Wnt/Btrc signaling pathway. These findings suggest that LncPGCR may exert a facilitating function and play a key role in PGC' development.

Epigenetic modification can regulate the differential expression of $L n c P G C R$. Previous studies have shown that histone acetylation can change the structure of nucleosomes, keep the chromatin development open, and promote the binding of transcription factors to chromosomal DNA, which is conducive to gene transcription and expression [33]. What is interesting to us is that the transcription factor TCF7L2 can target the regulation of LncPGCR expression. Transcription factor 7-like-2 (TCF7L2), also known as T-cell transcription factor 4 (TCF-4), is a transcription factor containing a DNA-binding domain [34]. Previous studies 
have shown that TCF7L2 is an important component of the Wnt signaling pathway [35]. Moreover, our previous studies have verified that Wnt signaling pathways are involved in regulating the differentiation of ESCs to SSCs [19]. It was also found that TCF7L2 can affect the development of PGCs as a key downstream transcription factor of the Wnt signaling pathway(data not published), suggesting that $L n c P G C R$ is regulated by WNT signals to participate in the development of PGCs.

LncRNA can be used as a molecular sponge of endogenous miRNA, and its molecular regulatory mechanism through binding to miRNA is the ceRNA mechanism [36,37]. This mechanism has received attention since it was proposed and it represents a new model of gene expression regulation. Wang found that linc-ROR can regulate human ESC maintenance and differentiation, and the subsequent sequence and function analysis found that because linc-ROR shared miRNA response elements with core transcription factors such as OCT4, SOX2, and NANOG, Linc-ROR acts as a sponge during ESC cell differentiation, preventing these core transcription factors from miRNA-mediated inhibition [38]. Here, our results suggest a novel model whereby LncPGCR promotes PGC development by acting as a ceRNA for gga-miR-6577-5p (Figure 5F).

In this study, we identified that Btrc is a potential target of gga-miR-6577-5 and LncPGCR. Btrc (also known as $\mathrm{SCF}^{\beta-\mathrm{TrCP}}$ ) is a key downstream gene of the WNT pathway. As a member of the F-box family, it encodes an F-box protein $(\beta-\operatorname{Tr} C P)$, and the coding protein $\beta$-TrCP of Btrc is one of the basic members of $\mathrm{SCF}^{\beta-\operatorname{TrCP}}$ [39]. In our research

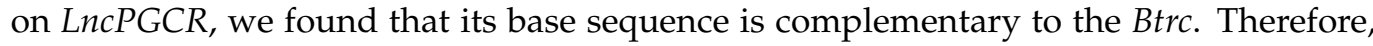
we speculate that $L n c P G C R$ may affect the expression of Btrc and ultimately affect the development of PGCs. Here, we confirmed that $L n c P G C R$ can promote the expression of Btrc. In addition, our results also demonstrated that gga-miR-6577-5 functioned as a suppressor of PGC development by directly targeting Btrc.

\section{Conclusions}

In sum, our results indicate that $L n c P G C R$ functions as a potential stimulator of PGCs development through the gga-miR-6577-5/Btrc axis. Our findings may offer a novel positive regulator and more efficient induction methods for PGCs development.

Supplementary Materials: The following are available online at https:/ / www.mdpi.com/2076-261 5/11/2/292/s1, Figure S1: LncPGCR regulated the expression of the totipotent gene Nanog and PGC marker genes to promote PGCs development (A). qRT-PCR of Nanog, Cvh, and C-kit expression after overexpression or knockdown with LncPGCR in vitro. ${ }^{*} p<0.05$, and ${ }^{* *} p<0.01$ compared with untreated ESCs. Values are means \pm SEM. $n=3$. (B). qRT-PCR of Nanog, Cvh, and C-kit expression after overexpression or knockdown with LncPGCR in vivo. ${ }^{*} p<0.05$, and ${ }^{* *} p<0.01$ compared with the untreated genital ridge. Values are means \pm SEM. $n=3$. (C). The proportion of PGCs-like cells $\left(\mathrm{Cvh}^{+}\right)$in the genital ridge on E4.5d detected by flow cytometry analysis. Table S1: LncPGCR promoter region different length PCR amplification primers, Table S2: Transcription factor TCF7L2 binding site mutation primer. Table S3: The transcript expression levels of PGCs lncRNA related to germ cell differentiation in ESCs, PGCs, and SSCs.

Author Contributions: J.J. (Jingyi Jiang), C.C., S.C., B.L., Y.Z., Q.Z. and G.C. conceived and designed the study and contributed to the acquisition, analysis, and interpretation of data; J.J. (Jingyi Jiang), C.C., X.Y., J.J. (Jing Jin), C.Z., X.S., J.S., and Q.Z. were involved in drafting the manuscript and revising it for important intellectual content. J.J. (Jingyi Jiang) and C.C. contributed equally to this work. All authors have read and agreed to the published version of the manuscript.

Funding: This work was funded by the Key Research and Development Program (2017YFE0108000), National Natural Science Foundation of China (31772582, 31872341 and 31572390), High-Level Talents Support Program of Yangzhou University, Jiangsu Science and Technology Project (Youth Fund): BK20180918; Natural Science Research Project of Jiangsu Higher Education Institutions: 18KJB230008.

Institutional Review Board Statement: All experimental procedures in the present study were reviewed and approved by the Institutional Animal Care and Use Committee of Yangzhou University (approval number: 151-2014). Procedures were performed in accordance with the Regulations for 
the Administration of Affairs Concerning Experimental Animals (Yangzhou University, China, 2012) and the Standards for the Administration of Experimental Practices (Jiangsu, China, 2008). We also confirm that the field studies did not involve endangered or protected species.

Informed Consent Statement: Not applicable.

Data Availability Statement: The data presented in this study are available on request from the corresponding author.

Acknowledgments: Thanks to the Poultry Institute of the Chinese Academy of Agricultural Sciences Experimental Poultry Farm for providing experimental materials.

Conflicts of Interest: The authors declare no conflict of interest.

\section{References}

1. Tsunekawa, N.; Naito, M.; Sakai, Y.; Nishida, T.; Noce, T. Isolation of chicken vasa homolog gene and tracing the origin of primordial germ cells. Development 2000, 127, 2741. [PubMed]

2. Saitou, M.; Barton, S.C.; Surani, M.A. A molecular programme for the specification of germ cell fate in mice. Nat. Cell Biol. 2002, 418, 293-300. [CrossRef] [PubMed]

3. Zhao, G.Q.; Garbers, D.L. Male Germ Cell Specification and Differentiation. Dev. Cell 2002, 2, 537-547. [CrossRef]

4. Ohinata, Y.; Payer, B.; O'Carroll, D.; Ancelin, K.; Ono, Y.; Sano, M.; Barton, S.C.; Obukhanych, T.; Nussenzweig, M.C.; Tarakhovsky, A.; et al. Blimp1 is a critical determinant of the germ cell lineage in mice. Nat. Cell Biol. 2005, 436, 207-213. [CrossRef] [PubMed]

5. Ancelin, K.; Lange, U.C.; Hajkova, P.; Schneider, R.J.; Bannister, A.J.; Kouzarides, T.; Surani, M.A. Blimp1 associates with Prmt5 and directs histone arginine methylation in mouse germ cells. Nat. Cell Biol. 2006, 8, 623-630. [CrossRef]

6. Kehler, J.; Tolkunova, E.; Koschorz, B.; Pesce, M.; Gentile, L.; Boiani, M.; Lomelí, H.; Nagy, A.; McLaughlin, K.J.; Schöler, H.R.; et al. Oct4 is required for primordial germ cell survival. EMBO Rep. 2004, 5, 1078-1083. [CrossRef] [PubMed]

7. Magnúsdóttir, E.; Surani, M.A. How to make a primordial germ cell. Deelopment 2013, 141, 245-252. [CrossRef]

8. Yamaji, M.; Seki, Y.; Kurimoto, K.; Yabuta, Y.; Yuasa, M.; Shigeta, M.; Yamanaka, K.; Ohinata, Y.; Saitou, M. Critical function of Prdm14 for the establishment of the germ cell lineage in mice. Nat. Genet. 2008, 40, 1016-1022. [CrossRef]

9. Kallayanee, C.; Svingen, T.; Ng, E.T.; Epp, T.; Spill, C.M.; Clark, C.; Cooper, H.; Koopman, P. Loss of Wnt5a Disrupts Primordial Germ Cell Migration and Male Sexual Development in Mice. Biol. Reprod. 2012, 86, 1-12.

10. de Sousa Lopes, S.M.C.; Roelen, B.A.J.; Monteiro, R.M.; Emmens, R.; Lin, H.Y.; Li, E.; Lawson, K.A.; Mummery, C.L. BMP signaling mediated by ALK2 in the visceral endoderm is necessary for the generation of primordial germ cells in the mouse embryo. Genes Dev. 2004, 18, 1838-1849. [CrossRef]

11. Margueron, R.; Trojer, P.; Reinberg, D. The key to development: Interpreting the histone code? Curr. Opin. Genet. Dev. 2005, 15, 163-176. [CrossRef] [PubMed]

12. Karagenc, L.; Petitte, J.N. Soluble factors and the emergence of chick primordial germ cells in vitro. Poult. Sci. 2000, 79, 80-85. [CrossRef] [PubMed]

13. Ying, Y.; Qi, X.; Zhao, G.-Q. Induction of primordial germ cells from murine epiblasts by synergistic action of BMP4 and BMP8B signaling pathways. Proc. Natl. Acad. Sci. USA 2001, 98, 7858-7862. [CrossRef] [PubMed]

14. Seisenberger, S.; Andrews, S.; Krueger, F.; Arand, J.; Walter, J.; Santos, F.; Popp, C.; Thienpont, B.; Dean, W.; Reik, W. The Dynamics of Genome-wide DNA Methylation Reprogramming in Mouse Primordial Germ Cells. Mol. Cell 2012, 48, 849-862. [CrossRef] [PubMed]

15. Sasaki, H.; Matsui, Y. Epigenetic events in mammalian germ-cell development: Reprogramming and beyond. Nat. Rev. Genet. 2008, 9, 129-140. [CrossRef]

16. Chong, S.; Whitelaw, E. Epigenetic germline inheritance. Curr. Opin. Genet. Dev. 2004, 14, 692-696. [CrossRef]

17. Saitou, M.; Kagiwada, S.; Kurimoto, K. Epigenetic reprogramming in mouse pre-implantation development and primordial germ cells. Development 2012, 139, 15-31. [CrossRef]

18. Wang, M.; Sun, C.; Li, D.; Li, T.; Zhang, W.; Jin, J.; Qi-Sheng, Z.; Ya-Ni, Z.; Guo-Hong, C.; Bi-Chun, L. Construction of RNA Interference Vector of Chicken(Gallus domesticus) FGF8 Gene and Its Effect on the Formation of PGCs. J. Agric. Biotechnol. 2018, 26, 1457-1466.

19. He, N.; Wang, Y.; Zhang, C.; Wang, M.; Wang, Y.; Zuo, Q.; Zhang, Y.; Li, B. Wnt signaling pathway regulates differentiation of chicken embryonic stem cells into spermatogonial stem cells via Wnt5a. J. Cell. Biochem. 2017, 119, 1689-1701. [CrossRef]

20. Zhang, C.; Wang, M.; Jin, J.; He, N.; Li, T.; Sun, X.; Cai, H.; Shu-Jian, Z.; Chang-Hua, S.; Qi-Sheng, Z.; et al. Construction of RNA Interference Vector of Hsd3 32 Gene and the Regulation of Chicken (Gallus gallus) ESCs Differentiation to PGCs. J. Agric. Biotechnol. 2018, 26, 1567-1576.

21. Bao, J.; Wu, J.; Schuster, A.S.; Hennig, G.W.; Yan, W. Expression profiling reveals developmentally regulated lncRNA repertoire in the mouse male germline. Biol. Reprod. 2013, 89, 107. [CrossRef] [PubMed]

22. Cesana, M.; Cacchiarelli, D.; Legnini, I.; Santini, T.; Sthandier, O.; Chinappi, M.; Tramontano, A.; Bozzoni, I. A Long Noncoding RNA Controls Muscle Differentiation by Functioning as a Competing Endogenous RNA. Cell 2011, 147, 358-369. [CrossRef] [PubMed] 
23. Tsai, M.-C.; Manor, O.; Wan, Y.; Mosammaparast, N.; Wang, J.K.; Lan, F.; Shi, Y.; Segal, E.; Chang, H.Y. Long Noncoding RNA as Modular Scaffold of Histone Modification Complexes. Science 2010, 329, 689-693. [CrossRef] [PubMed]

24. Guttman, M.; Garber, M.; Levin, J.Z.; Donaghey, J.; Robinson, J.T.; Adiconis, X.; Fan, L.; Koziol, M.J.; Gnirke, A.; Nusbaum, C.; et al. Ab initio reconstruction of cell type-specific transcriptomes in mouse reveals the conserved multi-exonic structure of lincRNAs. Nat. Biotechnol. 2010, 28, 503-510. [CrossRef]

25. Mohamed, J.S.; Gaughwin, P.M.; Lim, B.; Robson, P.; Lipovich, L. Conserved long noncoding RNAs transcriptionally regulated by Oct4 and Nanog modulate pluripotency in mouse embryonic stem cells. RNA 2009, 16, 324-337. [CrossRef]

26. Batista, P.J.; Chang, H.Y. Long Noncoding RNAs: Cellular Address Codes in Development and Disease. Cell 2013, $152,1298-1307$. [CrossRef]

27. Gong, N.; Teng, X.; Li, J.; Liangcd, X.-J. Antisense Oligonucleotide-Conjugated Nanostructure-Targeting lncRNA MALAT1 Inhibits Cancer Metastasis. ACS Appl. Mater. Interfaces 2018, 11, 37-42. [CrossRef]

28. Livak, K.J.; Schmittgen, T.D. Analysis of Relative Gene Expression Data using Real-Time Quantitative PCR. Method 2002, 25, 402-408. [CrossRef]

29. Minucci, S.; Pelicci, P.G. Histone deacetylase inhibitors and the promise of epigenetic (and more) treatments for cancer. Nat. Rev. Cancer 2006, 6, 38-51. [CrossRef]

30. Wang, X.; Wang, Q.; Wang, J.; Bai, P.; Shi, L.; Shen, W.; Zhou, M.; Zhou, X.; Zhang, Y.; Cai, M. Mit1 Transcription Factor Mediates Methanol Signaling and Regulates the Alcohol Oxidase 1 (AOX1) Promoter inPichia pastoris. J. Biol. Chem. 2016, 291, 6245-6261. [CrossRef]

31. Zheng, W.-P.; Flavell, R.A. The Transcription Factor GATA-3 Is Necessary and Sufficient for Th2 Cytokine Gene Expression in CD4 T Cells. J. Immunol. 2016, 196, 4426-4435. [CrossRef]

32. Salmena, L.; Poliseno, L.; Tay, Y.; Kats, L.; Pandolfi, P.P. A ceRNA Hypothesis: The Rosetta Stone of a Hidden RNA Language? Cell 2011, 146, 353-358. [CrossRef] [PubMed]

33. Nightingale, K.P.; Wellinger, R.E.; Sogo, J.M.; Becker, P.B. Histone acetylation facilitates RNA polymerase II transcription of the Drosophila hsp26 gene in chromatin. Embo J. 2014, 17, 2865-2876. [CrossRef]

34. Damcott, C.M.; Pollin, T.I.; Reinhart, L.J.; Ott, S.H.; Shen, H.; Silver, K.D.; Mitchell, B.D.; Shuldiner, A.R. Polymorphisms in the transcription factor 7-like 2 (TCF7L2) gene are associated with type 2 diabetes in the Amish: Replication and evidence for a role in both insulin secretion and insulin resistance. Diabetes 2006, 55, 2654-2659. [CrossRef] [PubMed]

35. Kwak, S.H.; Cho, Y.; Moon, M.K.; Kim, J.; Park, B.; Cheong, H.; Shin, H.-D.; Jang, H.; Kim, S.; Lee, H.; et al. Association of polymorphisms in the insulin-degrading enzyme gene with type 2 diabetes in the Korean population. Diabetes Res. Clin. Pr. 2008, 79, 284-290. [CrossRef] [PubMed]

36. Sun, M.; Nie, F.; Wang, Y.; Zhang, Z.; Hou, J.; He, D.; Xie, M.; Xu, L.; De, W.; Wang, Z.-X.; et al. LncRNA HOXA11-AS Promotes Proliferation and Invasion of Gastric Cancer by Scaffolding the Chromatin Modification Factors PRC2, LSD1, and DNMT1. Cancer Res. 2016, 76, 6299-6310. [CrossRef] [PubMed]

37. Song, X.; Cao, G.; Jing, L.; Lin, S.; Wang, X.; Zhang, J.; Wang, M.; Liu, W.; Lv, C. Analysing the relationship between lncRNA and protein-coding gene and the role of lncRNA as ceRNA in pulmonary fibrosis. J. Cell. Mol. Med. 2014, 18, 991-1003. [CrossRef]

38. Wang, Y.; Xu, Z.; Jiang, J.; Xu, C.; Kang, J.; Xiao, L.; Wu, M.; Xiong, J.; Guo, X.; Liu, H. Endogenous miRNA Sponge lincRNA-RoR Regulates Oct4, Nanog, and Sox2 in Human Embryonic Stem Cell Self-Renewal. Dev. Cell 2013, 25, 69-80. [CrossRef]

39. Camp, N. WTX is a novel regulator of ubiquitination in the Wnt/beta-catenin and KEAP1/NRF2 pathways. J. Anal. Psychol. 2012, $20,57-68$. 